\title{
Prevalence of Non-Fermenting Gram Negative Bacilli and their Antibiotic Sensitivity Pattern at a Tertiary Care Hospital in Tamilnadu, India
}

\author{
Sudha Krishnan, Priya Santharam*, N. Shanmugavadivoo and B. Usha \\ Department of Microbiology, Annapoorana medical college hospital, Salem, Tamilnadu, India \\ *Corresponding author
}

\begin{tabular}{|c|}
\hline Keywords \\
\hline $\begin{array}{l}\text { Non-fermenting gram } \\
\text { negative bacilli, } \\
\text { Clinical samples, } \\
\text { Antibiotic resistance }\end{array}$ \\
\hline Article Info \\
\hline $\begin{array}{l}\text { Accepted: } \\
24 \text { January } 2018 \\
\text { Available Online: } \\
10 \text { February } 2018\end{array}$ \\
\hline
\end{tabular}

A B S T R A C T

Non-fermenting gram negative bacilli (NFGNB) are known to account for nearly 12$16 \%$ all bacterial isolates from a clinical microbiology laboratory. The most common infections caused by these organisms were septicemia, pneumonia, urinary tract infections, surgical site infections, wound infections, osteomyelitis, etc. As multidrug resistances being very common and increasing among NFGNB and Pseudomonas and Acinetobacter being the most predominantly isolated NFGNB and its resistance towards colistin and imepenam type of antimicrobials is of major concern. To isolate, identify and characterize the prevalence of NFGNB along with their antimicrobial sensitivity pattern among the patients attending a tertiary care centre in Tamilnadu. A prospective study was conducted in our hospital for a period of two years from Jan2012 to Dec 2013. A total of 5052 clinical specimens were received during the above said period. Out of this 1699 were urine specimens, 315 were pus, 988 blood, 1470 respiratory samples which includes sputum and tracheal secretions, and 580 were other than the above mentioned samples (body fluids, stool, tissue biopsy, vaginal swabs etc). The isolates that showed non lactose fermenting (NLF) colonies on Mac conkey agar and failed to acidify the butts of triple sugar iron (TSI) agar were provisionally considered as NFGNB. Antimicrobial sensitivity was determined by Kirby Bauer disc diffusion method on Muller Hinton agar (MHA). Antibiotic discs were placed and plates were incubated at $37^{\circ} \mathrm{C}$ for $18-24 \mathrm{hrs}$. Results were interpreted in accordance with central laboratory standards institute (CLSI) guidelines. In our study out of 5052 clinical samples 517 samples had shown positive for non-fermenting gram negative bacilli with a prevalence of $10.2 \%$. Pseudomonas aeruginosa (53.9\%) was found to be the most common organism isolated from the clinical samples followed by Acinetobacter baumanni (36.7\%). The antibiotic sensitivity pattern varies for different clinical samples but colisitin and imipenam had shown the maximum sensitivity pattern for all the clinical samples. The sensitivity pattern for gentamicin, ceftazidime and ciprofloxacin was in the range of $30-70 \%$ which means highest resistance was seen with these antimicrobials. It is important to establish the clinical relevance of the isolated NFGB, before are considered as pathogens to avoid unnecessary usage of antibiotics and emergence of drug-resistant strains. 


\section{Introduction}

The non-fermentative gram-negative bacilli (NFGB) are group organisms with characteristics of aerobic, non-spore forming, gram negative bacilli which either utilize carbohydrates as a source of energy nor degrade them through metabolic pathways other than fermentation. (Winn et al., 2006) These organisms are most commonly recovered from hospital environment, which would cause device related infections and they are often resistant to disinfectants and are considered to be more hazardous as it has the potential to spread from patient-to-patient either via fomites or through the hands of the medical personnel. (Steinberg and Rio, 2005; Quinn, 1998) Now recently these nonfermenting bacteria which are associated with different nosocomial infections are becoming increasingly resistant to the commonly used antibiotics and are also known to produce extended spectrum $\beta$ - lactamases and metallo $\beta$ - lactamases. (McGowan, 2006) NFGNB are known to account for nearly $12-16 \%$ all bacterial isolates from a clinical microbiology laboratory. The most common infections caused by these organisms were septicemia, pneumonia, urinary tract infections, surgical site infections, wound infections, osteomyelitis, etc. (Bergogne-Berezin and Towner, 1996; Mehta et al., 2001) These heterogeneous group includes organisms like Pseudomonas spp, Acinetobacter spp, Alkaligenes spp, Stenotrophomonas maltophilia, Burkholderia cepaciacomplex (BCC). Currently Pseudomonas aeruginosa and Acinetobacter baumannii are the most commonly isolated non-fermenters pathogenic for humans. Infections caused by other species are relatively infrequent (Fass et al., 1996).

Few studies were done to identify the risk factors for NFGNB infections and it identified immunosuppression (oncology patients on cytotoxic therapy/radiotherapy, organ transplant patients and even patients with AIDS), neutropenia, mechanical ventilation, cystic fibrosis, indwelling catheters, invasive diagnostic and therapeutic procedures were the common risk factors for acquiring NFGNB infections and prolonged hospital stay, use of broad spectrum antibiotic and underlying host factors were found to be the best predictors of the outcome of this infection (Quinn, 1998).

Multidrug resistance is very common and increasing among NFGNB. Carbapenem resistance among Pseudomonas and Acinetobacter is of major concern. Carbapenemase activity in A. baumannii is mainly due to carbapenem-hydrolyzing class $\mathrm{D}$ b-lactamases (CHDLs) which is very much specific for this species. These enzymes belong to 3 unrelated groups of clavulanic acid resistant b-lactamases represented by OXA-23, OXA-24, and OXA-58 that can be either plasmid or chromosomally encoded (Poirel and Nordmann, 2006).

In case of $P$. aeruginosa the dominant mechanism of carbapenem resistance is loss of carbapenem specific porin OprD2 (Quinn et al., 1988). There are number of strains which had been identified and proved showing resistance to essentially most of the commonly used antibiotics. Recently few studies conducted in India had identified the antimicrobial susceptibility pattern for NFGNB (Samanta et al., 2011; Gautam et al., 2009; Malini et al., 2009).

\section{Aim}

To isolate, identify and characterize the prevalence of NFGNB along with their antimicrobial sensitivity pattern among the patients attending a tertiary care centre in Tamilnadu.

\section{Materials and Methods}


A Prospective study was conducted in our hospital for a period of two years from Jan 2012-December 2013. The pathogens data were extracted from Culture and sensitivity registers which was audited for correctness by Head of Microbiology department on weekly basis. The bed strength of our hospital is 550 with 30 beds in the intensive care unit. A total of 5052 clinical specimens were received during the above said period. Out of this 1699 were urine specimens, 315 were pus, 988 blood, 1470 respiratory samples which includes sputum and tracheal secretions, and 580 were other than the above mentioned samples (body fluids, stool, tissue biopsy, vaginal swabs etc).

All the samples were plated on blood agar (BA) and MacConkey's agar (MA) and incubated at $37^{\circ} \mathrm{C}$ for 48 hours before being reported as sterile. The isolates that showed non lactose fermenting (NLF) colonies on MacConkey agar and failed to acidify the butts of triple sugar iron (TSI) agar were provisionally considered as NFGNB and they were further identified by using a standard protocol for identification. The characters assessed were gram staining morphology, motility (by hanging drop), catalase test, oxidase test, citrate utilization, urea hydrolysis, hemolysis on 5\% sheep blood agar, growth on $6.5 \% \mathrm{NaCl}$, nitrate reduction, pigment production, indole production, lysine and ornithine decarboxylation, arginine dihydrolase test, growth at $40^{\circ} \mathrm{C}$ and $42^{\circ} \mathrm{C}$, oxidation of $1 \%$ glucose, lactose, sucrose, maltose, mannitol, xylose (Hugh and Leifson's medium), growth on $10 \%$ lactose agar and gelatin liquefaction test.

Antimicrobial sensitivity was determined by Kirby Bauer disc diffusion method on Muller Hinton agar (MHA). Briefly a suspension of each isolate was made so that the turbidity was equal to $0.5 \mathrm{McF}$ arland standards and then plated as a lawn culture onto MHA. Antibiotic discs were placed and plates were incubated at $37^{\circ} \mathrm{C}$ for $18-24 \mathrm{hrs}$. Results were interpreted in accordance with central laboratory standards institute (CLSI) guidelines. Escherichia coli ATCC 25922 and Pseudomonas aeruginosa ATCC 27853 were used as control strains. Antibiotics which were used for testing the susceptibility are Amikacin, Gentamycin, Ciprofloxacin, Ofloxacin, piperacillin, PIT, aztreonam, tobramycin, ceftazidime, colistin, Imepenam, meropenam and norfloxacin.

\section{Results and Discussion}

In our study out of 5052 clinical samples 517 samples had shown positive for nonfermenting gram negative bacilli with a prevalence of $10.2 \%$. Table 1 shows the total number of non-fermenting gram negative bacilli isolated from different clinical samples. It is seen from the table that Pseudomonas and Acinetobacter are the two prevalent Non fermenting gram negative bacilli which were isolated and among the Pseudomonas genus $P$. aeruginosa and $P$. fluorescens are the two common species which were isolated and among the Acinetobacter it was A.baumannii and $A$. Iwoffii were the two common species which were isolated. Among all the four organism Pseudomonas aeruginosa (53.9\%) was found to be the most common organism isolated from the clinical samples followed by Acinetobacter baumanni (36.7\%). Whereas the prevalence of Pseudomonas fluorescens and Acinetobacter iwoffii was 6\% and 3\% respectively. Pseudomonas aeroginosa was found to be more common among the pus and urine samples and less common in blood samples, whereas Acinetobacter baumannii was found to be more common in blood samples and respiratory tract specimens and least common among the samples taken from pus. Table 2 shows the antibiotic sensitivity pattern among the Pseudomonas species from the different clinical samples collected. It is observed from the table that the antibiotic sensitivity pattern varies for different clinical samples but colisitin and Imipenam had shown 
the maximum sensitivity pattern for all the clinical samples. Apart from colistin and imipenam, piperacillin and tazobactum combination had shown the next highest sensitivity pattern among the various clinical samples. Piperacillin when used alone had shown the sensitivity pattern only between 50 $-65 \%$. Aztreonam is the next antimicrobial with a maximum sensitivity pattern ranging between $65 \%$ - $80 \%$. The sensitivity pattern for gentamicin, ceftazidime and ciprofloxacin was in the range of $30-70 \%$ which means highest resistance was seen with these antimicrobials. Norfloxacin was sensitive only for urine sample with a sensitivity of only $37 \%$. The antibiotic sensitivity pattern among the Acinetobacter species from the different clinical samples collected were shown in Table 3. It is inferred from the table that the sensitivity pattern for Acinetobacter was almost similar to that of the Pseudomonas organism except for norfloxacin antimicrobial which had shown a sensitivity pattern of $70 \%$ in the urine samples.

Non-fermenters are ubiquitous in environment. Although frequently they are considered as commensals or contaminants, the pathogenic potential of NFGNB has been established beyond doubt by their frequent isolation from clinical materials and their association with certain dreadful diseases (Winn et al., 2006; Prashanth and Badrinath, 2004). The available data suggests that NFGNB are remarkable microorganisms because of their epidemiological complexity, propensity to cause outbreaks of infection and antimicrobial resistance (Rahbar and Hajia, 2006; Boroumand et al., 2007; Taherikalani et al., 2008). They are now considered as the most important nosocomial pathogens especially among the immune compromised hosts for causing variety of infections. Resistance to antimicrobials has become more common among NFGNB and the resistance has now extended to all commonly used antibiotics. Multi drug resistance among these organisms makes the treatment more difficult and expensive (Kharangate et al., 2001).

Studies carried out by different researchers have reported varied isolation rates. In the present study the prevalence of NFGNB among the clinical samples was $10.2 \%$ and this was almost similar to the results of a study from Chandigarh (Taneja et al., 2003) where NFGNB were isolated in $10 \%$ of clinical samples. Contradicting to our study a study from Amritsar (Sidhu et al., 2010) reported a very high isolation rate of $45.9 \%$ and another study from Bangalore reported it to be $21.80 \%$ (Kumari et al., 2007). A study from Saudi Arabia (Eltahawy and Khalaf, 2001) had shown the prevalence as $16 \%$ and a study from Kolar, Karnataka (Malini et al., 2009). reported NFGNB to be isolated only in $4.5 \%$ of clinical samples and similarly a study from Brazil had also reported a very low isolation rate of only about $2.18 \%$ (Bruno et al., 2011). Pseudomonas was found to be commonest non fermenter in all of these studies followed by Acinetobacter and this is in concordance to our finding and also the most common species among Pseudomonas was aeroginosa and among Acinetobacter it is baumannii.

In our study, highest number of the NFGNB isolates were from respiratory samples, similar to the observations made by others (Taneja et al., 2003; Mishra et al., 1986). NFGNB were commonly involved in wound infections resulting from road traffic accidents and chronic non-healing ulcers. The clinical conditions associated with NFGNB infection in our study included surgical site infection (SSI), ventilator-associated pneumonia (VAP), urinary tract infection (UTI), septicemia and chronic nonhealing ulcer because of endocrinopathy like diabetes mellitus. $P$. aeruginosa and A. baumannii were more commonly isolated from respiratory samples, followed by pus and urine. 
Table.1 Total number of non-fermenting gram negative bacilli isolated from Different clinical samples

\begin{tabular}{|l|l|l|l|l|l|l|}
\hline $\begin{array}{l}\text { Name of the } \\
\text { organism }\end{array}$ & Pus & Blood & Urine & $\begin{array}{l}\text { Respiratory } \\
\text { tract }\end{array}$ & Others & Total \\
\hline P.aeruginosa & $60(75 \%)$ & $18(42.8 \%)$ & $42(57.5 \%)$ & $142(48.6 \%)$ & $17(56.6 \%)$ & $279(53.9 \%)$ \\
\hline A.baumannii & $14(17.5 \%)$ & $21(50 \%)$ & $24(32.8 \%)$ & $121(41.4 \%)$ & $10(33.3 \%)$ & $190(36.7 \%)$ \\
\hline P.fluorescens & $4(5 \%)$ & $1(2.3 \%)$ & $4(5.4 \%)$ & $21(7.1 \%)$ & $2(6.6 \%)$ & $32(6.1 \%)$ \\
\hline A.Iwoffii & $2(2.5 \%)$ & $2(4.7 \%)$ & $3(4.1 \%)$ & $8(2.7 \%)$ & $1(3.3 \%)$ & $16(3 \%)$ \\
\hline Total & $80(100 \%)$ & $42(100 \%)$ & $73(100 \%)$ & $292(100 \%)$ & $30(100 \%)$ & $517(100 \%)$ \\
\hline
\end{tabular}

Table.2 Antibiotic sensitivity pattern among the Pseudomonas species from the different clinical samples collected

\begin{tabular}{|l|l|l|l|l|l|}
\hline Antibiotics & Pus $(\mathbf{n}=\mathbf{6 4})$ & $\begin{array}{l}\text { Blood } \\
(\mathbf{n}=\mathbf{1 9})\end{array}$ & Urine $(\mathbf{n = 4 6})$ & $\begin{array}{l}\text { Respiratory } \\
\text { tract }(\mathbf{n}=\mathbf{1 6 3})\end{array}$ & $\begin{array}{l}\text { Others } \\
(\mathbf{n}=\mathbf{1 9})\end{array}$ \\
\hline Amikacin & $44(68.7 \%)$ & $15(78.9 \%)$ & $23(50 \%)$ & $93(57 \%)$ & $14(73.6 \%)$ \\
\hline Gentamicin & $34(53.1 \%)$ & $14(73.6 \%)$ & $12(26 \%)$ & $93(57 \%)$ & $12(63.1 \%)$ \\
\hline Ceftazidime & $34(53.1 \%)$ & $12(63.1 \%)$ & $29(63 \%)$ & $106(65 \%)$ & $13(68.4 \%)$ \\
\hline Ciprofloxacin & $46(71.8 \%)$ & $12(63.1 \%)$ & $17(36.9 \%)$ & $120(73.6 \%)$ & $12(63.1 \%)$ \\
\hline Pipericillin & $43(67.1 \%)$ & $9(47.3 \%)$ & $23(50 \%)$ & $108(66.2 \%)$ & $10(52.6 \%)$ \\
\hline PIT & $53(82.8 \%)$ & $18(94.7 \%)$ & $41(89.1 \%)$ & $132(80.9 \%)$ & $16(84.2 \%)$ \\
\hline Aztreonam & $41(64 \%)$ & $15(78.9 \%)$ & $34(73.9 \%)$ & $128(78.5 \%)$ & $15(78.9 \%)$ \\
\hline Tobramycin & $40(62.5 \%)$ & $16(84.2 \%)$ & $18(39.1 \%)$ & $93(57 \%)$ & $14(73.6 \%)$ \\
\hline Colistin & $58(90.6 \%)$ & $19(100 \%)$ & $46(100 \%)$ & $149(91.4 \%)$ & $18(94.7 \%)$ \\
\hline Imepenam & $58(90.6 \%)$ & $19(100 \%)$ & $46(100 \%)$ & $163(100 \%)$ & $18(94.7 \%)$ \\
\hline Norfloxacin & 0 & 0 & $17(36.9 \%)$ & 0 & 0
\end{tabular}

Table.3 Antibiotic sensitivity pattern among the Acinetobacter species from the different clinical samples collected

\begin{tabular}{|l|l|l|l|l|l|}
\hline Antibiotics & Pus $(\mathbf{n = 1 6 )}$ & $\begin{array}{l}\text { Blood } \\
(\mathbf{n = 2 3})\end{array}$ & Urine $(\mathbf{n = 2 7})$ & $\begin{array}{l}\text { Respiratory } \\
\text { tract }(\mathbf{n = 1 2 9})\end{array}$ & $\begin{array}{l}\text { Others } \\
(\mathbf{n = 1 1})\end{array}$ \\
\hline Amikacin & $10(62.5 \%)$ & $14(60.8 \%)$ & $14(51.8 \%)$ & $58(44.9 \%)$ & $8(72.7 \%)$ \\
\hline Gentamicin & $7(43.7 \%)$ & $10(43.4 \%)$ & $12(44.4 \%)$ & $36(27.9 \%)$ & $10(90.9 \%)$ \\
\hline Ceftazidime & $6(37.5 \%)$ & $8(34.7 \%)$ & $14(51.8 \%)$ & $36(27.9 \%)$ & $6(54.5 \%)$ \\
\hline Ciprofloxacin & $7(43.7 \%)$ & $15(65.2 \%)$ & $13(48.1 \%)$ & $36(27.9 \%)$ & $9(81.8 \%)$ \\
\hline Pipericillin & $7(43.7 \%)$ & $13(56.5 \%)$ & $14(51.8 \%)$ & $58(44.9 \%)$ & $5(45.4 \%)$ \\
\hline PIT & $15(93.7 \%)$ & $22(95.6 \%)$ & $25(92.5 \%)$ & $82(63.5 \%)$ & $11(100 \%)$ \\
\hline Aztreonam & $13(81.2 \%)$ & $20(86.9 \%)$ & $18(66.6 \%)$ & $53(41 \%)$ & $11(100 \%)$ \\
\hline Tobramycin & $13(81.2 \%)$ & $15(65.2 \%)$ & $17(62.9 \%)$ & $64(49.6 \%)$ & $11(100 \%)$ \\
\hline Colistin & $16(100 \%)$ & $21(91.3 \%)$ & $27(100 \%)$ & $112(86.8 \%)$ & $11(100 \%)$ \\
\hline Imepenam & $16(100 \%)$ & $22(95.6 \%)$ & $26(96.2 \%)$ & $129(100 \%)$ & $11(100 \%)$ \\
\hline Norfloxacin & 0 & 0 & $19(70.3 \%)$ & 0 & 0
\end{tabular}


Our study is in concordance with reports of other authors for multi-drug resistance among the $P$. aeruginosa (Takeyama et al., 2002; Jombo et al., 2008). High degree of resistance to almost all the routinely used antibiotics was seen and this finding is in line with the study from Chandigarh (Taneja et al., 2003). Though imipenem showed good activity to all the NFGNB, but emerging resistance to this group of drug is of major concern. Previous studies by other authors also have reported carbapenem resistance among NFGNB. (Taneja et al., 2003; Gladstone et al., 2005) In the present study only 5\% of Acinetobacter species and $8 \%$ of Pseudomonas species were imipenem resistant and this was in contrast to the findings of Gladstone et al., from Tamil Nadu and Joseph et al., from Pondicherry who have reported the same to be $12.2 \%$ and $50 \%$ respectively (Taneja et al., 2003; Gladstone et al., 2005). In our study, Acinetobacter strains percentage sensitivity for Colistin and Imipenem was $95.6 \%$ and $98.3 \%$ respectively. Imipenem monotherapy have also been proved effective in many studies (Sidhu et al., 2010).

The strains of Acinetobacter species showed higher rate of resistance to ciprofloxacin, amikacin, ceftazidime and piperacillin in a study in Bangalore which is almost in par with the present study (Sinha et al., 2007). Resistance to 3rd generation Cephalosporin, Ceftazidime showed $38 \%$ in Pseudomonas and 58\% among Acenitobacter and it was found to be higher than the studies done by Kumarietal and Mishra et al., which had reported the resistance in the range of $35-$ $40 \%$ for both Pseudomonas and Acinetobacter (Mishra et al., 1986; Kumari et al., 2007).

Pseudomonas showed (45\%) resistance to Gentamicin which is concordant with (Murugan et al., 2010) who also reported $(42.8 \%)$ resistance to Gentamicin. In the present study Ciprofloxacin resistance to
Pseudomonas is (39\%) which is very much lower than (Deepak Juyal et al., 2013) who had reported the prevalence of resistance as $73.7 \%$.

The antibiotic susceptibility patterns may change with time and may vary from hospital to hospital. Susceptibility patterns may be altered due to resistance transfer and mutant selection from indiscriminate and excessive use of antibiotics. Furthermore, most of our patients came from rural areas without much exposure to antibiotics. Differences in susceptibility could be attributed to these above mentioned factors.

Non-fermenter gram-negative bacilli though regarded as contaminants are important bacteria causing both hospital- and community-acquired infection. $P$. aeruginosa and $A$. baumannii were the most common NFGB isolated in our study. They have been associated with UTI, septicemia, SSI, VAP and other chronic wound infection and most of them were multidrug-resistant. $P$. aeruginosa has shown good sensitivity to colistin, imipenem, amikacin and cefoperazone/sulbactum combination. $A$. baumannii shows good sensitivity to colistin, imipenem and amikacin. Prompt identification of NFGB upto the species level along with monitoring of their susceptibility patterns are important for proper management of the infection caused by them.

It is also important to establish the clinical relevance of the isolated NFGB, before they can be considered as pathogens to avoid unnecessary usage of antibiotics and emergence of drug-resistant strains. Continued awareness of the need to maintain good housekeeping, equipment decontamination, strict attention to hand washing and isolation procedures are the measures necessary to control the previously unabated spread of these organisms 


\section{References}

Bergogne-Berezin E, and Towner KJ. Acinetobacter spp. as Nosocomial Pathogens: Microbiological, Clinical and Epidemiological Features. ClinMicrobiol Rev 1996 Apr; 9(2): 148-65.

Boroumand MA, Esfahanifard P, Saadat S, Sheihkvatan M, Hekmatyazdi S, Saremi M, et al., A report of Pseudomonas aeruginosa antibiotic resistance from a multicenter study in Iran. Indian J Med Microbiol 2007; 25:435-6.

Bruno D, Nishino MK, Priore WN, Remus PR, do Carmo AA, Stefanello VB, et al., Prevalence of Gram-negative nonfermenters patients in Porto Alegre-RS. JornalBrasileiro de Patologia e Medicina Laboratorial 2011; 47:529-34.

Deepak Juyal, Rajat Prakash, Shamanth A Shanakarnarayan, Munesh Sharma, Vikrant Negi, Neelam Sharma. Prevalence of nonfermenting gram negative bacilli and their in vitro susceptibility pattern in a tertiary care hospital of Uttarakhand: A study from foothills of Himalayas. 2013. VoI 2 (2) Page: 108-112.

Eltahawy AT, and Khalaf RM. Antibiotic resistance among gram-negative non fermentative bacteria at a teaching hospital in Saudi Arabia. J Chemother 2001; 13:260-4.

Fass RJ, Barnishan J, Solomon MC, Ayers LW. In vitro activities of quinolones, beta-lactams, tobramycin, and trimethoprimsulfamethoxazole against non-fermentative gram-negative bacilli. Antimicrob Agents Chemother 1996; 40:1412-8.

Gautam V, Ray P, Vandamme P, Chatterjee SS, Das A, Sharma K, et al., Identification of lysine positive non-fermenting gram negative bacilli (Stenotrophomonas maltophilia and Burkholderiacepacia complex). Indian J Med Microbiol 2009; 27:128-33.

Gladstone P, Rajendran P, Brahmadathan KN. Incidence of carbapenem resistant nonfermenting gram negative bacilli from patients with respiratory infections in the intensive care units. Indian J Med Microbiol 2005; 23:189-91.
In: Winn W Jr, Allen S, Janda W, Koneman E, Procop G, Schreckenberger $\mathrm{P}$, et al., editors. Non-fermenting Gram negative bacilli. In: Koneman's color Atlas and textbook of Diagnostic Microbiology. 6th ed. USA: Lippincott Williams and Wilkins Company; 2006. p. 305-91.

Jombo AG, Jonah P, Ayeni AJ. Multidrug resistant Pseudomonas aeruginosa in a contemporary Medical Practice: Findings from urinary isolates at a Nigerian University Teaching Hospital. Niger J PhysiolSci 2008; 23:105-9.

Kharangate NV, Pinto MJ, Rodrigues S, Verenkar MP. Characterization of non-fermenters from clinical samples. J Assoc Physicians India $2001 ;$ 49:324-6.

Kumari HB, Nagarathna S, Chandramuki A. Antimicrobial resistance pattern among aerobic gram-negative bacilli of lower respiratory tract specimens of intensive care unit patients in a neurocentre. Indian $\mathrm{J}$ Chest Dis Allied Sci 2007; 49(1):19-22.

Kumari HB, Nagarathna S, Chandramuki A. Antimicrobial resistance pattern among aerobic gram-negative bacilli of lower respiratory tract specimens of intensive care unit patients in a neurocentre. Indian $\mathrm{J}$ Chest Dis Allied Sci 2007; 49(1):19-22.

Malini A, Deepa EK, Gokul BN, Prasad SR. Nonfermenting gram-negative bacilli infections in a tertiary care hospital in Kolar, Karnataka. J Lab Physicians 2009; 1:62-6.

Malini A, Deepa EK, Gokul BN, Prasad SR. Nonfermenting gram-negative bacilli infections in a tertiary care hospital in Kolar, Karnataka. J Lab Physicians 2009; $1: 62-6$.

McGowan JE Jr. Resistance in non-fermenting gram negative bacteria: multidrug resistance to the maximum Am $\mathrm{J}$ Infect Control 2006; 34(5 Suppl 1): S29-37; discussion S64-73.

Mehta M, Punia JN, Joshi RM. Antibiotic resistance in Pseudomonas aeruginosa strains isolated from various clinical specimens- a retrospective study. Indian J Med Microbiol 2001; 19(4):232. 
Mishra B, Bhujwala RA, Shriniwas. Nonfermenters in human infections. Indian $\mathbf{J}$ Med Res 1986; 83: 561-6.

Murugan S., Bakkiya Lakshmi R., Umadevi P. and Mani K.R. Prevalence and Antimicrobial susceptibility Pattern of Metallo $\quad \beta$-lactamase producing Pseudomonas aeruginosa in Diabetic Foot Infection. Int. J. of Microbiological Research 2010. 1(30), 123-128.

Poirel L, and Nordmann P. Carbapenem resistance in Acinetobacter baumannii: Mechanisms and epidemiology. ClinMicrobiol Infect 2006; 12:826-36.

Prashanth K, and Badrinath S. In vitro susceptibility pattern of Acinetobacter species to commonly used cephalosporins, quinolones, and aminoglycosides. Indian $\mathbf{J}$ Med Microbiol 2004; 22:97-103.

Quinn JP, Studemeister AE, DiVincenzo CA, Lerner SA. Resistance to imipenem in Pseudomonas aeruginosa: Clinical experience and biochemical mechanisms. Rev Infect Dis 1988; 10:892-8.

Quinn JP. Clinical problems posed by multi resistant non-fermenting gram negative pathogens. Clinl Infect Dis 1998; 27(Suppl 1):S117-24.

Quinn JP. Clinical problems posed by multiresistant non-fermenting gramnegative pathogens. Clin Infect Dis 1998; 27Suppl 1:S117-24.

Rahbar M, and Hajia M. Detection and quantitation of the etiologic agents of ventilator-associated pneumonia in endotracheal tube aspirates from patients in Iran. Infect Control HospEpidemiol 2006; 27:884-5.

Samanta P, Gautam V, Thapar R, Ray P. Emerging resistance of non-fermenting gram negative bacilli in a tertiary care centre. Indian J PatholMicrobiol 2011; 54:666-7.

Sidhu S, Arora U, Devi P. Prevalence of nonfermentative gram negative bacilli in seriously ill patients with bacteraemia. JK Science 2010; 12: 168-71.

Sinha M, Srinivasa H, Macaden R. Antibiotic resistance profile and extended spectrum beta-lactamase (ESBL) production in Acinetobacter species. Indian $\mathrm{J}$ Med Res 2007; 126(1):63-7.

Steinberg JP, and Rio DC. Other Gram-negative and Gram variable bacilli. In: Principles and Practice of Infectious Diseases. 6th edition, Vol. 2, Mandell GL, Bennett JE, Dolin R (Eds.), Elsevier Publication: Philadelphia, USA 2005:p.2751-68.

Taherikalani M, Etemadi G, Geliani KN, Fatollahzadeh B, Soroush S, Feizabadi MM. Emergence of multi and pan-drug resistance Acinetobacter baumannii carrying blaOXA-type -carbapenemase genes among burn patients in Tehran, Iran. Saudi Med J 2008; 29:623-4.

Takeyama K, Kunishima Y, Matsukawa M, Takahashi S, Hirose T, Kobayashi N, et al., Multidrug-resistant Pseudomonas aeruginosa isolated from the urine of patients with urinary tract infection. J Infect Chemother 2002; 8:59-63.

Taneja N, Maharwal S, Sharma M. Imipenem resistance in nonfermenters causing nosocomial urinary tract infections. Indian J Med Sci 2003; 57(7):294-9.

Winn W Jr, Allen S, Janda W, Koenman E, Procop G, Schreckenberger P, et al., (Eds.). Non-fermenting Gram negative bacilli. In Koneman's Color Atlas and Textbook of Diagnostic Microbiology. 6th edition, Lippincott Williams and Wilkins Company: USA 2006: Pp. 305-91.

\section{How to cite this article:}

Sudha Krishnan, Priya Santharam, N. Shanmugavadivoo and Usha, B. 2018. Prevalence of Non-Fermenting Gram Negative Bacilli and Their Antibiotic Sensitivity Pattern at a Tertiary Care Hospital in Tamilnadu. Int.J.Curr.Microbiol.App.Sci. 7(02): 2751-2758. doi: https://doi.org/10.20546/ijcmas.2018.702.335 